\title{
NCAPH regulates gastric cancer progression through DNA damage response
}

\author{
Yan WANG ${ }^{1,2, *}$, Jun-Qiang LI ${ }^{1, *}$, Zhi-Liang YANG ${ }^{1,2, *}$, Long WANG ${ }^{1}$, Jun-Chang ZHANG ${ }^{1}$, Yong-Fang SUN ${ }^{1}$, Zi-Yue LI' ${ }^{1}$, Long QIN ${ }^{1,2, *}$ \\ ${ }^{1}$ Lanzhou University Second Hospital, Lanzhou, China; ${ }^{2}$ Huazhi Tiancheng Biotechnology, Lanzhou, China \\ ${ }^{*}$ Correspondence: ery_qin@lzu.edu.cn \\ ${ }^{*}$ Contributed equally to this work.
}

Received June 7, 2021 / Accepted August 9, 2021

\begin{abstract}
Gastric cancer (GC) is one of the most common devastating and deadly malignancies of the gastrointestinal tract in the world. GLOBOCAN data analysis showed that GC accounted for approximately 1,033,000 new cases of cancer and 78,200 deaths in 2018. Nonstructural maintenance of chromosomes (non-SMC) condensin I complex subunit H (NCAPH) is a regulatory subunit that encodes the non-SMC condensin I complex. Previous studies have demonstrated that NCAPH is highly expressed in multiple cancers. This study aimed to explore the function and potential mechanism of NCAPH in GC. Our study showed that NCAPH expression was significantly upregulated in The Cancer Genome Atlas (TCGA) and Oncomine datasets. Quantitative real-time polymerase chain reaction and western blotting were used to detect NCAPH expression in GC and paracarcinoma tissues. Cell Counting Kit-8 (CCK-8) and colony formation assays were used to examine cell proliferation. Cell scratch and Transwell invasion assays were performed to assess cell migration. In addition, western blotting was used to detect the expression of proteins related to the cell cycle, DNA damage repair, and epithelialmesenchymal transition (EMT). Flow cytometry was applied for cell cycle and apoptosis detection. A xenograft model was employed to assess the effect of NCAPH in vivo. The results demonstrated that NCAPH expression was significantly increased in GC tissue samples and cell lines. Knockout of NCAPH notably inhibited cell proliferation, cell migration, cell invasion, cell cycle progression, and tumor growth in vitro and in vivo, and induced the G1-phase cell cycle arrest by regulating the DNA damage response. In addition, knockout of NCAPH promoted cell apoptosis and regulated the expression of EMT-related proteins. The results indicate that the knockout of NCAPH in GC cells inhibits proliferation and metastasis via the DNA damage response in vitro and in vivo. NCAPH plays an important role in GC and may be a potential therapeutic target for GC treatment.
\end{abstract}

Key words: NCAPH, gastric cancer, proliferation, DNA damage response

Gastric cancer (GC) is one of the most common fatal malignant tumors, has a poor prognosis worldwide, and is a major threat to human health $[1,2]$. GLOBOCAN data analysis showed that GC accounted for approximately 1,033,000 new cases of cancer and 78,200 deaths in 2018 [3]. The incidence and mortality of cancer in China are increasing, and 67,910 new GC cases and 49,800 GC-related deaths were reported in 2015 . Unfortunately, the survival rate of GC is very low. Studies show that the five-year survival rate is only 28\% [4]. Furthermore, most of the GC patients are diagnosed at an advanced stage, which increases the difficulty of the treatment process $[5,6]$. Although considerable progress and achievements have been made in the treatment of $\mathrm{GC}$, the rapid proliferation of tumors is still the biggest obstacle for the clinical treatment of GC, and cancer cell mitosis is a critical process in proliferation. Thus, there is an urgent need to find a novel target for GC treatment that focuses on the mitotic process.

Condensins are protein complexes that promote chromosome assembly and segregation during mitosis and meiosis [7]. Eukaryotes have two distinct types of condensin complexes: condensin I and II. Some studies have shown that condensin I can arrange loops of chromatin, while condensin II is required for the rigidity of an initial chromosome axis $[8,9]$. Gene expression profiling of GC samples and adjacent normal samples from 8 patients was performed using an mRNA microarray; condensing I complex subunit (NCAPH) was identified as a potential candidate target gene for the treatment of GC. NCAPH is located on human chromosome $2 \mathrm{q} 11.2$ and is also known as BRRN1 [10]. It is involved in the stability of the condensin protein complexes and plays an important role in the precise segregation of sister chroma- 
tids during mitosis $[7,11]$. Chen et al. reported that NCAPH expression is significantly higher in GC tissues than in normal tissues [12]. Previous studies have shown that downregulation of NCAPH significantly inhibits cell proliferation and induces apoptosis and cell cycle arrest in pancreatic cancer cells, which is consistent with the results of colon cancer, prostate cancer, and lung cancer [13-15]. Although NCAPH has been identified as a biomarker of multiple cancers, the detailed mechanisms remain unclear.

In this study, we explored the expression and distribution of NCAPH in human GC. In addition, we found that NCAPH knockout inhibited GC cell proliferation in vitro and repressed tumor growth in vivo. These data suggested that NCAPH is an important contributor to the pathogenesis of GC.

\section{Patients and methods}

Ethical approval. The study was approved by the ethics committee of Lanzhou University Second Hospital (approval number: D2020-36), and all patients signed an informed consent form. The animal study was approved by the ethics committee of Lanzhou University Second Hospital.

Gene expression data analysis. The expression level of the NCAPH gene in different tumors was analyzed using The Cancer Genome TCGA (https://tcga-data.nci.nih.gov/tcga) and Oncomine databases (https://www.oncomine.org/), and these data are publicly available.

Patients and samples. The subjects were GC cancer patients who were surgically treated in our general surgery center. Samples of GC tissues and corresponding adjacent normal tissues were collected from the patients. The tissue samples were stored at $-80^{\circ} \mathrm{C}$. All tissue samples were divided into two parts: one part was processed for quantitative real-time PCR (qRT-PCR) and the other part was used for protein extraction.

Cell culture. Human GC cell lines (AGS, HGC27, and MKN45) were purchased from the Chinese Academy of Sciences, and HEK293T cells were obtained from the American Type Tissue Culture (ATCC). All cell lines were grown in DMEM supplemented with $10 \%$ fetal bovine serum and cultured at $37^{\circ} \mathrm{C}$ with $5 \% \mathrm{CO}_{2}$ in a humidified incubator.

Lentivirus preparation and infection. NCAPH was knocked out by guide RNA (gRNA), which was designed based on the CRISPR/Cas9 system. The plasmid was constructed using the Gibson assembly cloning method [16]. Lentiviral vectors containing gRNA, pSPAX2, and pMD2G were inserted into HEK293T cells by co-transfection. Then, lentiviruses were harvested to infect GC cells (AGS, HGC27, and MKN45), which were selected with puromycin. NCAPH knockout was validated using western blots.

Quantitative real-time fluorescent PCR (qRT-PCR). Total RNA extraction of GC tumor tissues and cell lines was performed using the AG RNAex Pro RNA reagent. Total RNA was reverse transcribed into cDNA using the Transcriptor
First Strand cDNA Synthesis Kit, and iTap Universal SYBR Green Premix Supermix was used for real-time PCR amplification. Target genes were detected on a Roche LightCycler 96 system. The qRT-PCR forward primer sequence for NCAPH was GTCCTCGAAGACTTTCCTCAGA, and the reverse primer sequence was TGAAATGTCAATACTCCTGCTGG; the glyceraldehyde-3-phosphate dehydrogenase (GAPDH) forward primer sequence was GAAGGCTGGGGCTCATTT, and the reverse primer sequence was CAGGAGGCATTGCTGATGAT.

Western blotting. Protein was extracted from GC tumor tissues, and cell line proteins were extracted using radioimmunoprecipitation assay (RIPA) lysis buffer. The protein concentration was measured with the BCA Protein Assay Kit. First, protein samples were separated by polyacrylamide sodium dodecyl sulfate gel electrophoresis and then electrotransferred to polyvinylidene difluoride membranes. The membranes were incubated in TBST $(10 \mathrm{mM}$ Tris- $\mathrm{HCl}$, $150 \mathrm{mM} \mathrm{NaCl}, 0.1 \%$ Tween 20, pH 7.4) with 5\% skim milk for $1 \mathrm{~h}$ at room temperature and then incubated with primary antibodies overnight at $4^{\circ} \mathrm{C}$. After washing with TBST, the corresponding secondary antibody was incubated with the membranes at room temperature for $2 \mathrm{~h}$. Finally, the protein was detected using NcmECL Ultra. The primary and secondary antibodies for western blotting were as follows: anti-NCAPH (Proteintech Group, 11515-1-AP, 1:1000), anti-Cyclin A2 (Proteintech Group, 18202-1-AP, 1:1000), anti-Cyclin B1 (Proteintech Group, 55004-1-AP, 1:1000), anti-Cyclin D1 (Proteintech Group, 26939-1-AP, 1:1000), anti-CtIP (Cell Signaling, D76F7, 1:1000), anti-P95NBS1 (Cell Signaling, D6J5I, 1:1000), anti-RAD54 (Cell Signaling, D4W3Z, 1:1000), anti-N-cadherin (Abcam, ab98952, 1:1000), anti-vitronectin (Sigma, 045M4795V, 1:1000), antivimentin (Proteintech Group, 60330-I-Ig, 1:1000), antismad2 (Proteintech Group, 12570-1-AP, 1:1000) and $\beta$-actin (Proteintech Group, 20536-1-AP, 1:1000), goat anti-mouse IgG $(\mathrm{H}+\mathrm{L}), \mathrm{HRP}$ (Proteintech Group, SA00001-1, 1:5000), goat anti-Rabbit IgG $(\mathrm{H}+\mathrm{L})$, HRP (Proteintech Group, SA00001-2, 1:5000).

Cell proliferation and colony formation assay. Cell proliferation was measured using the Cell Counting Kit (CCK-8) assay. NCAPH knockout GC cell lines and control cells (AGS and HGC27) were seeded in 96-well plates at a density of $3 \times 10^{3}$ cells/well and cultured for $24,48,72,96$, and $120 \mathrm{~h}$. Then, one-tenth the volume of the CCK- 8 reagent was added, and the plate was incubated at $37^{\circ} \mathrm{C}$ for $1 \mathrm{~h}$. The absorbance was measured at $450 \mathrm{~nm}$. Furthermore, NCAPH knockout cell lines (AGS and HGC27) and controls were seeded in $35 \mathrm{~mm}$ dishes for the colony formation assay. After 14 days in culture, the cells were fixed with $4 \%$ methanol, stained with $0.1 \%$ crystal violet, and counted.

Cell scratch and Transwell invasion assay. Cell migration was measured by a scratch wound-healing assay. A total of $3 \times 10^{5}$ cells were seeded in 6 -well plates, the cell layers were scratched using a $200 \mu \mathrm{l}$ pipette to make parallel 
linear wounds, and the debris was removed by washing with phosphate-buffered saline (PBS). After 24 h of culture, the cell migration areas were measured, and then the migration rate was calculated. The migration and invasion abilities of cells were assessed using Transwell plates precoated with Matrigel. Two hundred microliters of serum-free DMEM with $4 \times 10^{4}$ cells were seeded in each Transwell chamber, and the lower chamber contained a medium with $10 \%$ FBS. After $24-48 \mathrm{~h}$ of culture, cells in the lower chambers were fixed with $4 \%$ methanol and stained with $0.1 \%$ crystal violet.

Flow cytometry analysis. Flow cytometry was applied for the cell cycle and apoptosis detection. The cells were collected by centrifugation $\left(300 \times \mathrm{g} / \mathrm{min}, 5 \mathrm{~min}, 4^{\circ} \mathrm{C}\right)$ and washed twice with precooled PBS. One part was used for cell cycle detection, and the other was used for cell apoptosis detection. For one part, the harvested cells were fixed in 75\% ethanol at $4{ }^{\circ} \mathrm{C}$ overnight, and stained with propidium (PI); then, cell cycle distribution was analyzed by flow cytometry. Cells in the other part were stained with Annexin V-FITC and PI Staining Solution at RT for $10 \mathrm{~min}$ and then analyzed apoptosis by flow cytometry.

Animal experiments. Twelve NSG mice (5-6 weeks old; an equal mix of males and females) were randomly divided into an experimental group and a control group (WT). NCAPH knockout MKN45 cells and control cells treated with Matrigel alone were subcutaneously injected into NSG mice. The body weight and tumor volume of NSG mice were measured every three days using a Vernier caliper. Tumor volume was determined with the formula: Volume $=$ width $^{2} / 2$ $\times$ (length).

Statistical analysis. All experimental data are presented as the mean \pm standard deviation (SD) deviation of at least three replicate experiments. Statistical analysis and data plotting were performed using GraphPad Prism version 8. A paired t-test was used to compare NCAPH expression between precancerous and tumor tissues. A p-value $<0.05$ was considered statistically significant.

\section{Results}

NCAPH was significantly upregulated in GC tissues. First, we found that NCAPH was highly expressed in GC tissues by gene chip analysis (Figure 1A). We further assessed the expression of NCAPH in GC versus noncancerous tissues in TCGA database and found that NCAPH expression was upregulated in GC tissues (Figure 1B). Consistent with this observation, NCAPH was significantly upregulated in cancer of the alimentary tract based on the Oncomine database (Figure 1C). We detected the expression of NCAPH mRNA in 11 normal GC tissues by qRT-PCR. NCAPH mRNA expression was significantly higher in GC tissues than in normal tissues (Figure 1D). Western blot analysis also demonstrated that the protein level of NCAPH was markedly higher in GC tissues than in the corresponding adjacent normal tissues (Figure 1E). The immunohistochemistry staining results showed that NCAPH expression was significantly higher in GC tissues than in paracarcinoma tissues and that NCAPH was mainly localized to the nucleus in human GC cells (Figure 1F). It was revealed by Kaplan-Meier analyses that overexpression of NCAPH was not associated with the survival rate in GC (Figure $1 \mathrm{G}$ ). The above results suggested that overexpression of NCAPH is associated with the clinical development of GC.

NCAPH was significantly upregulated in GC cells. Western blot analysis demonstrated that the protein level of NCAPH was markedly higher in five GC cell lines than in GES cells (Figure 2A). At the same time, the mRNA expression levels of NCAPH were determined using qRT-PCR, which showed that mRNA expression levels were significantly upregulated in GC cell lines (Figure 2B). To address the biological function of NCAPH in the progression of GC, we designed a specific gRNA targeting the NCAPH gene and assessed its knockout effect on the GC cell lines AGS, HGC27, and MKN45. Western blot analysis verified that gRNA significantly inhibited NCAPH expression in cells (Figure 2C). Next, we further studied the effect of NCAPH on cell function.

NCAPH knockout suppressed the proliferation, migration, and invasion of GC cells. To evaluate the effect of NCAPH knockout on the biological function of GC cells, we employed CCK-8, colony formation, Transwell, and invasion assays using NCAPH gRNA specifically targeting NCAPH. The CCK- 8 assay showed that NCAPH knockout led to a remarkable reduction of proliferation (Figure 2D) in AGS and HGC27 cells compared with that in the control group. Moreover, compared to the control condition, knockout of NCAPH significantly inhibited colony formation in AGS and HGC27 cells (Figure 2F). Furthermore, NCAPH knockout significantly suppressed cell migration (Figure 2E) and invasion (Figure 2G) in the two GC cell lines. These results indicate that NCAPH plays an essential role in the cellular function of GC cells.

NCAPH knockout suppressed the cell cycle and DNA damage response. The functions and locations of NCAPH in the cells were determined by the gene set enrichment analysis and immunofluorescence. Upregulated gene enrichment analysis revealed that NCAPH was mainly enriched in the cell cycle and DNA replication (Figures 3A, 3B). Moreover, the results showed that the NCAPH protein was located mainly in the nucleus (Figure 3C). As shown in Figures 3D-3G, knockout of NCAPH induced the G1 phase cell cycle arrest, and the proportion of cells in the S and G2 phases obviously decreased in the NCAPH knockout group in AGS and HGC27 cells. Our data indicated that the knockout of NCAPH significantly inhibited proteins related to the cell cycle (cyclin A2 and cyclin B1) (Figure 3H). Previous studies have indicated that the NCAPH is closely related to the DNA damage response. To elucidate whether NCAPH influences the biological function of cancer cells via regulation of the DNA damage response, we analyzed the effects of NCAPH on 


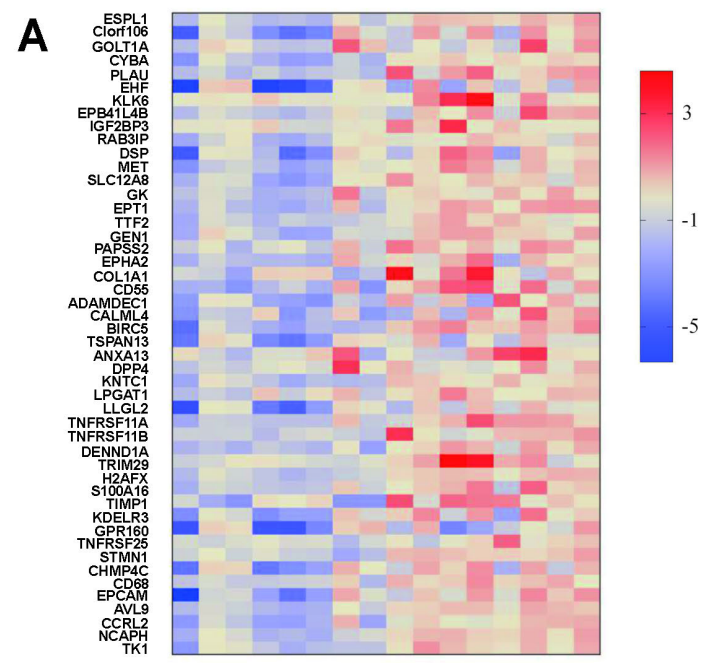

B

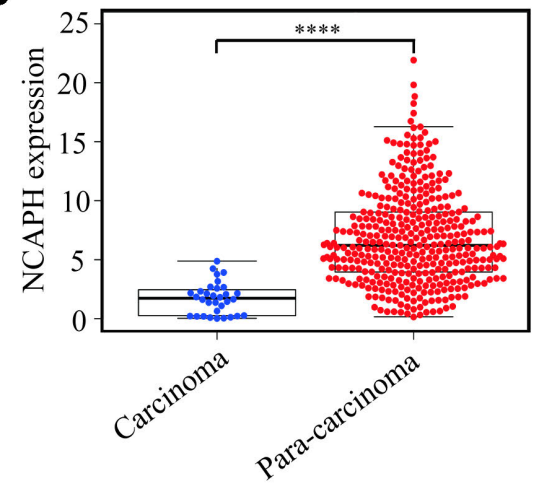

C
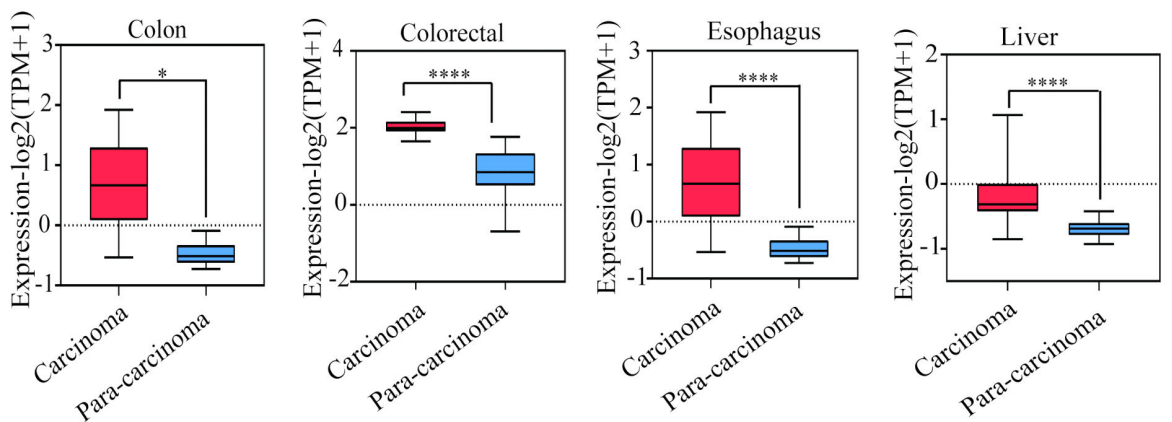

D

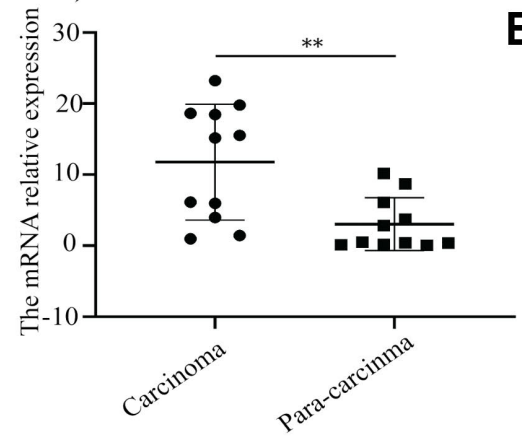

E
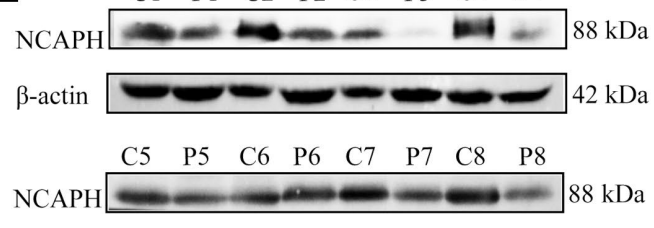

$\beta$-actin
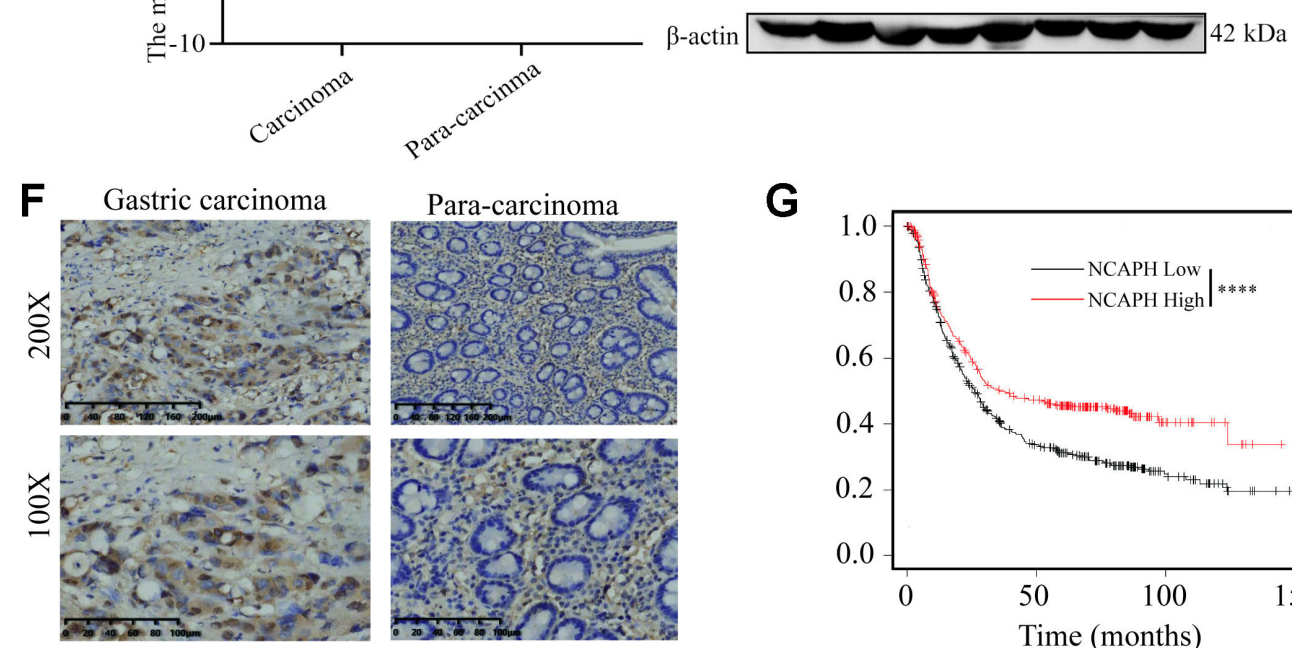

Para-carcinoma

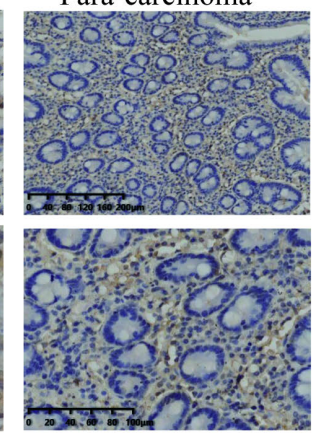

G

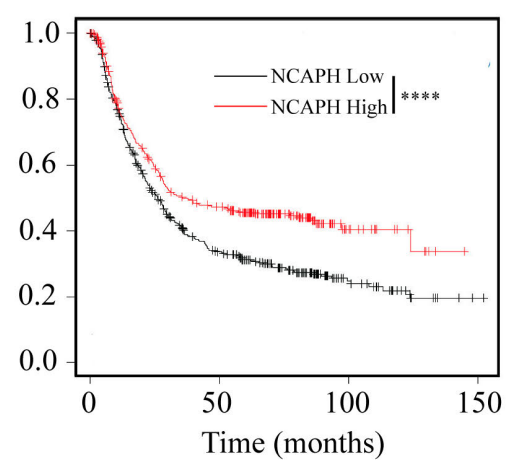

Figure 1. NCAPH is upregulated in multiple cancers. A) Highly expressed genes from the gastric samples were analyzed with a gene expression microarray. B) NCAPH is highly expressed in GC tissues. ${ }^{*} \mathrm{p}<0.01,{ }^{* *} \mathrm{p}<0.01,{ }^{* * *} \mathrm{p}<0.001,{ }^{* * * *} \mathrm{p}<0.0001$ in all figures. C) NCAPH is highly expressed in multiple cancers. D) The mRNA expression level of NCAPH in GC tissues and paracarcinoma tissues. E) The protein expression level of NCAPH in GC tissues and paracarcinoma tissues. F) Representative images of immunohistochemical staining for NCAPH in GC tissues and paracarcinoma tissues. G) The association between NCAPH expression and overall survival rate in GC patients. 
A
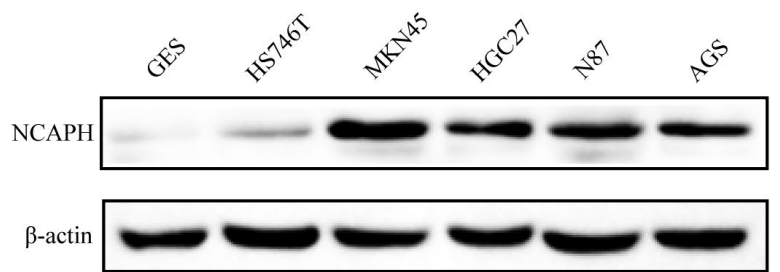

C

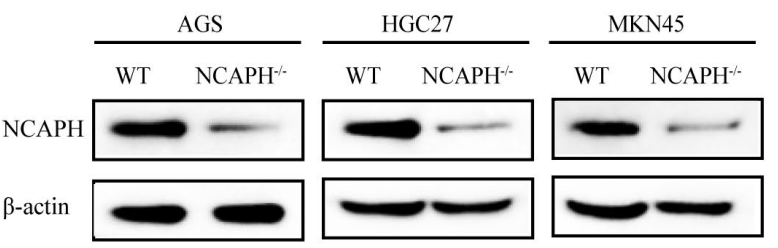

B

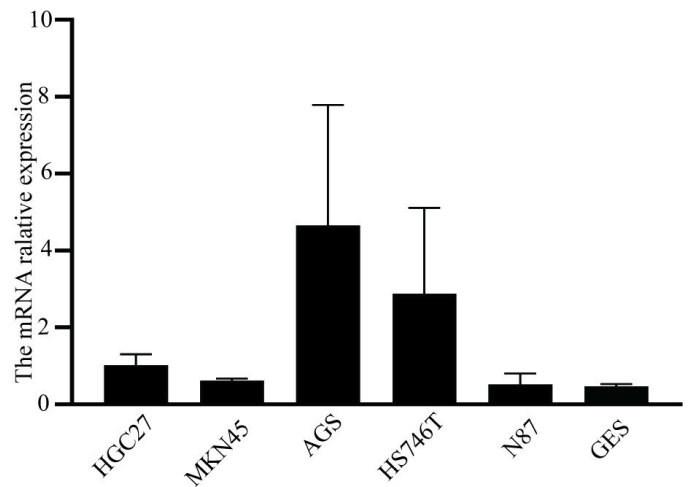

D
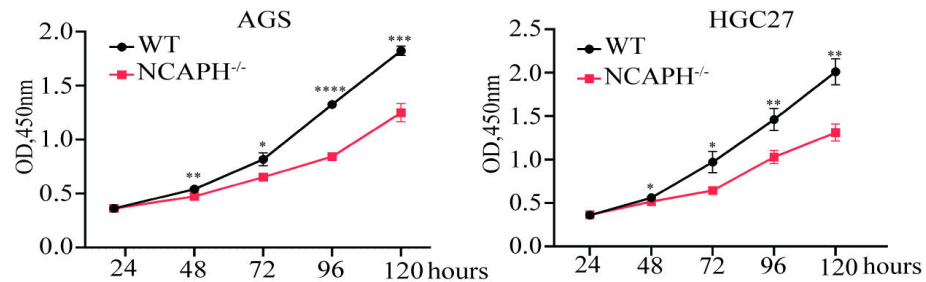

E
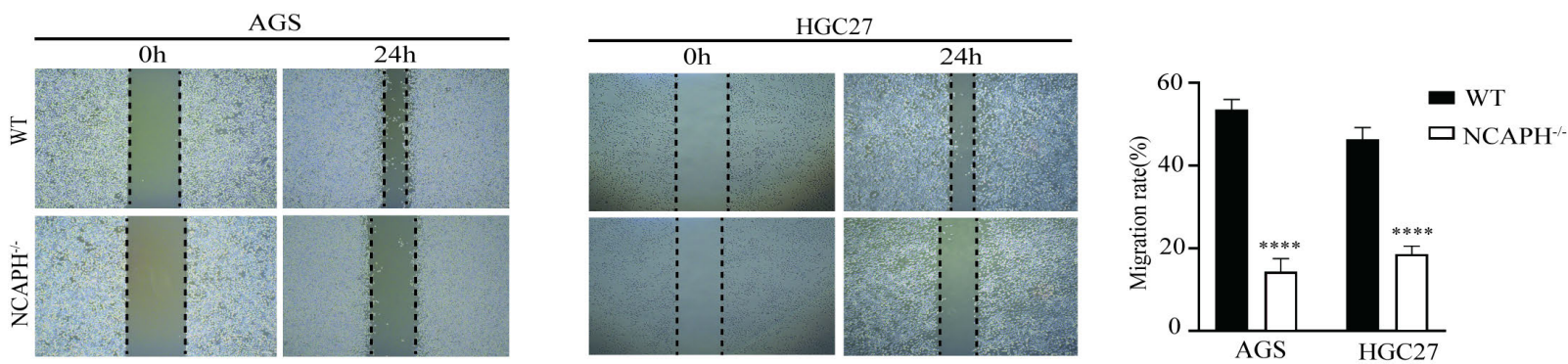

$\mathbf{F}$
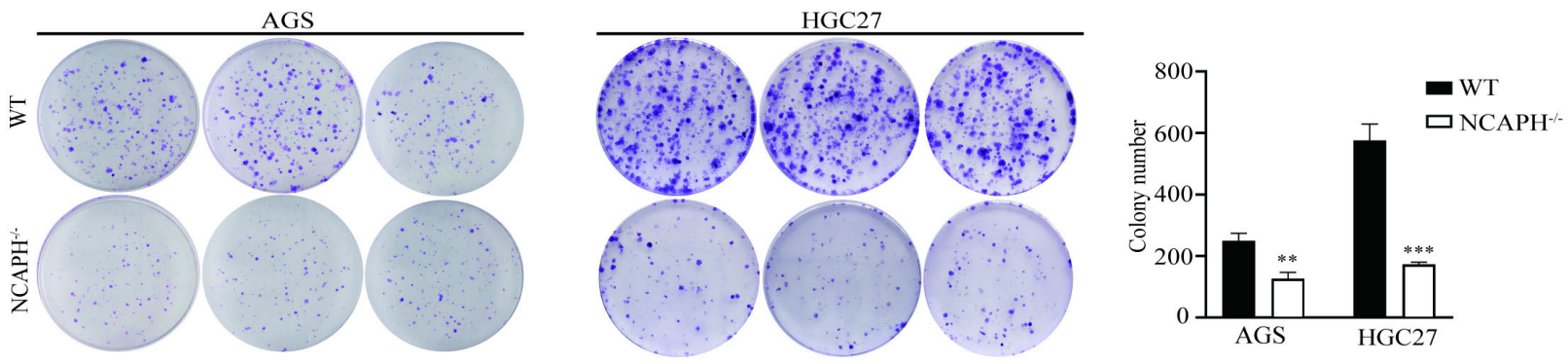

G

AGS
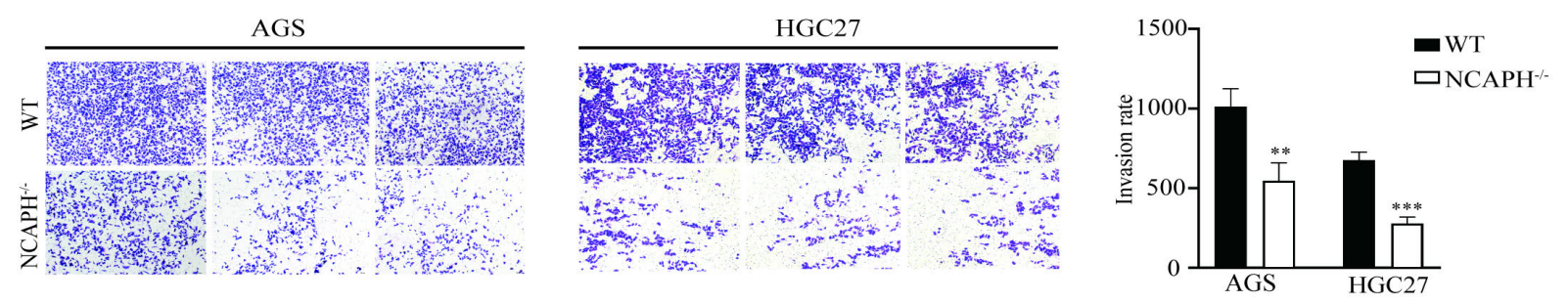

Figure 2. NCAPH knockout significantly inhibited the proliferation, migration, and invasion of AGS and HGC27 cells. A) The protein expression level of NCAPH in GC cells. B) The mRNA expression level of NCAPH in GC cells. C) The knockout efficiency of NCAPH was validated by western blotting. D) Proliferation of the AGS and HGC27 cell lines was measured by a CCK-8 assay. E) Representative image and quantification of wound-healing assays in the AGS and HGC27 cell lines. F) NCAPH knockout suppressed the colony formation in the AGS and HGC27 cell lines. G) Representative image and quantification of Transwell invasion assays in the AGS and HGC27 cell lines. 
A
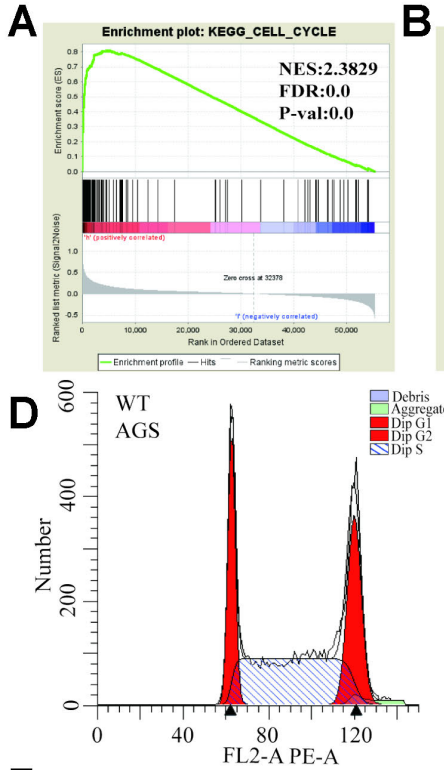

F

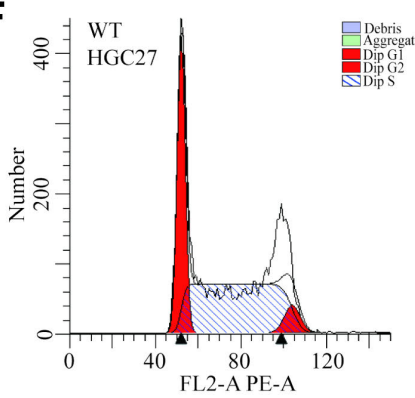

B
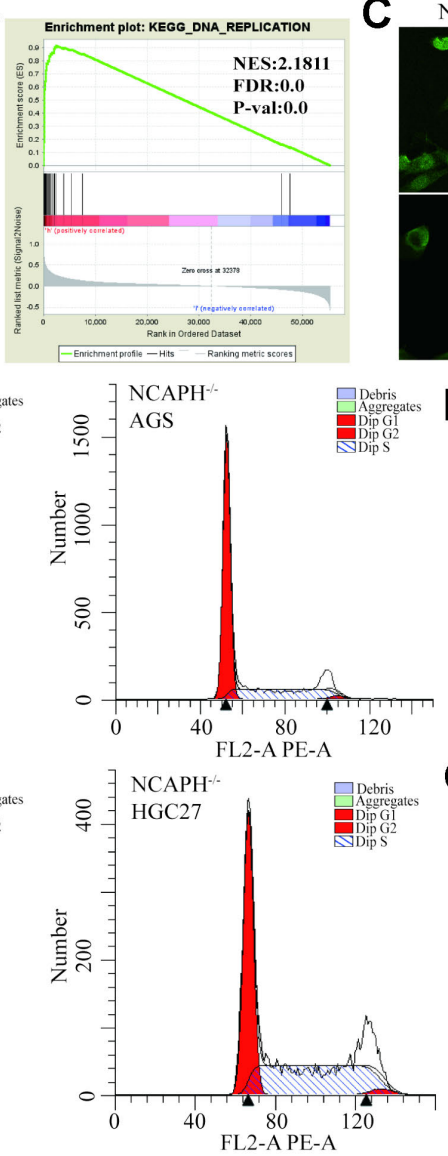

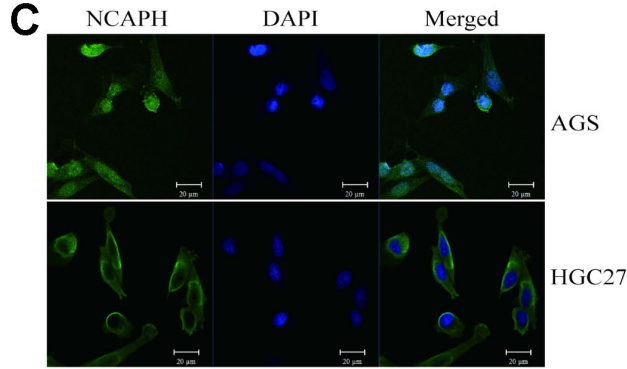

E

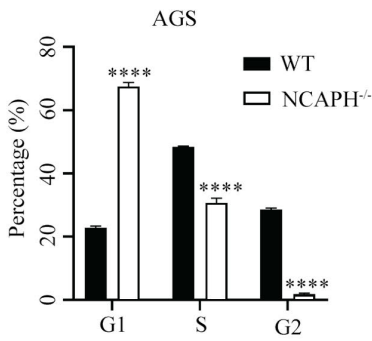

G

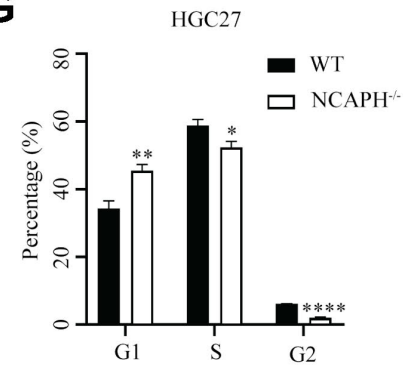

$\mathrm{H}$

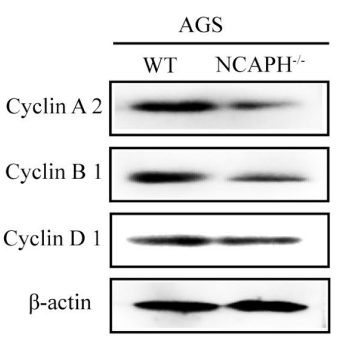

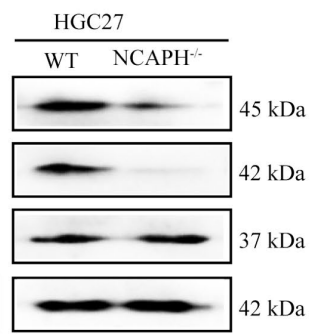

I
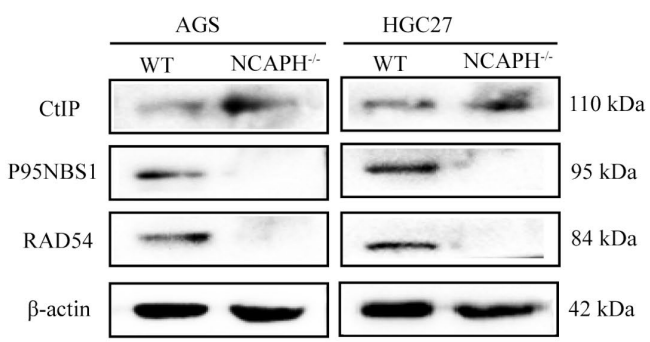

J
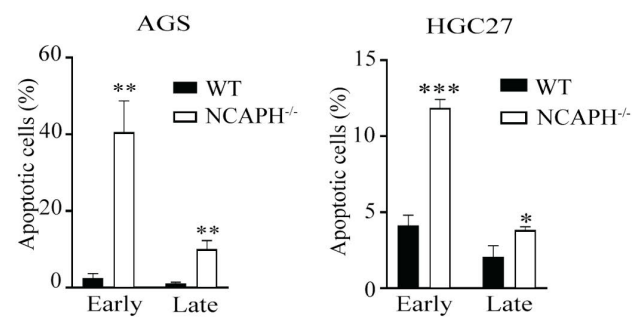

K

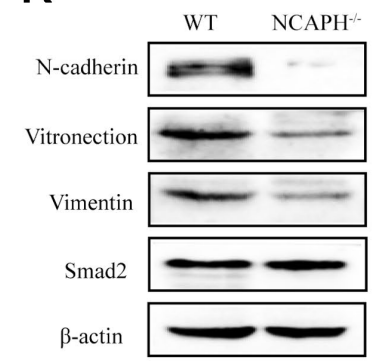

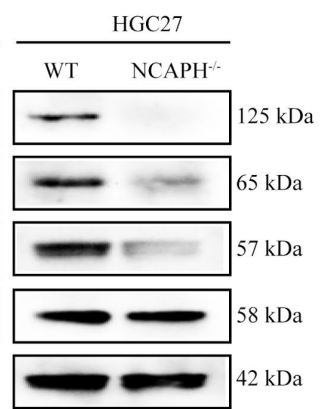

Figure 3. NCAPH knockout suppressed the cell cycle arrest and inhibited DNA damage repair proteins expression. The gene set enrichment analysis revealed that NCAPH was enriched in the cell cycle (A) and DNA replication (B). C) Immunofluorescence staining of the cellular localization of NCAPH (green) in AGS and HGC27 cells. DAPI (blue) was used to stain the nuclei. Scale bars $20 \mu \mathrm{m}$. D-G) The percentages of cells in each cell cycle phase were detected using flow cytometry. The proportion of cells in the G1 phase significantly increased while the proportions of cells in the $S$ and G2 phases obviously decreased in AGS and HGC27 cells. H, I, K) Western blot analysis showed that NCAPH knockout inhibited the expression of proteins related to the cell cycle, DNA damage repair, and EMT. J) NCAPH knockout promoted early and late stages AGS and HGC27 cell apoptosis, as determined by flow cytometry. 
the expression levels of DNA damage repair proteins in AGS and HGC27 cells by western blotting. The results showed that compared with that in the control group, expression of the DNA damage repair proteins P95NBS1 and RAD54 was significantly weakened by NCAPH knockout in AGS and HGC27 cells (Figure 3I). However, the CtIP signal did not change significantly when NCAPH was knocked out in AGS and HGC27 cells (Figure 3I). In addition, EMT-related proteins play crucial roles in EMT, thus serving as markers in the assessment of EMT. Our studies showed that knockout of NCAPH promoted early-stage and late-stage apoptosis and suppressed the expression of EMT-related proteins in AGS cells and HGC27 cells (Figures 3J, 3K).

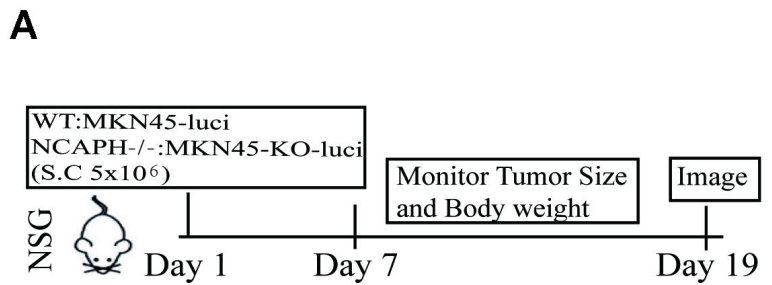

B

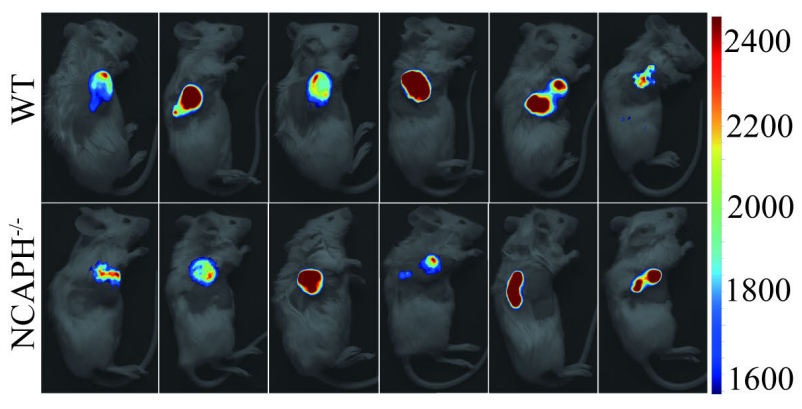

E

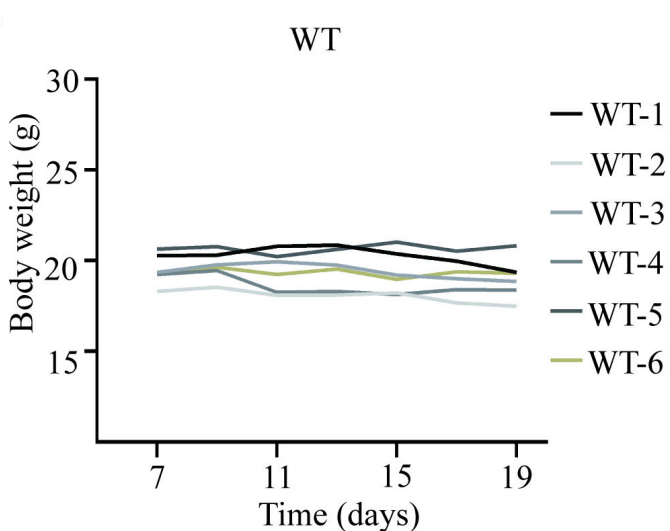

C

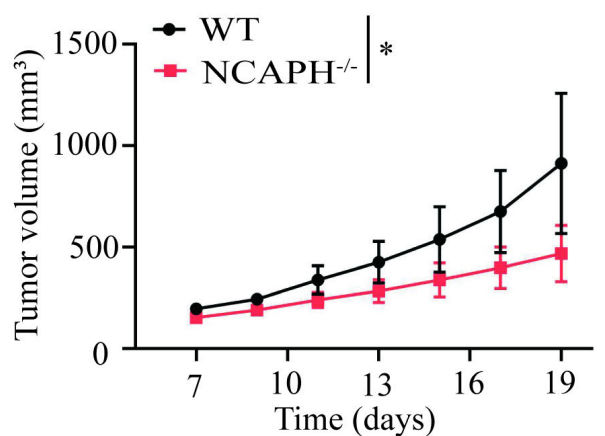

D

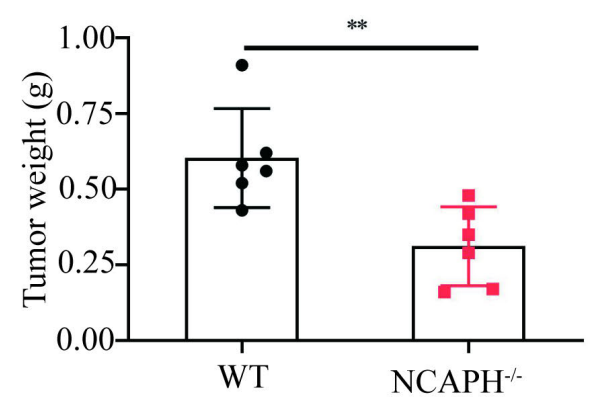

NCAPH knockout inhibited subcutaneous gastric tumorigenesis in NSG mice. We conducted in vivo animal experiments (Figure 4A) to further evaluate the effect of knocking out NCAPH using a subcutaneous tumorigenesis model. NCPAH knockout significantly reduced tumor volume and tumor weight compared with those in the control group (Figures 4B-4D). Moreover, the knockout of $\mathrm{NCAPH}$ had no impact on the bodyweight of mice (Figures $4 \mathrm{E}, 4 \mathrm{~F})$. In all the analyses we conducted, the sex of the NSG mice showed no significant effect on the variables. The animal study results suggested that NCAPH knockout inhibits the subcutaneous tumorigenicity of MKN45 cells in NSG mice.

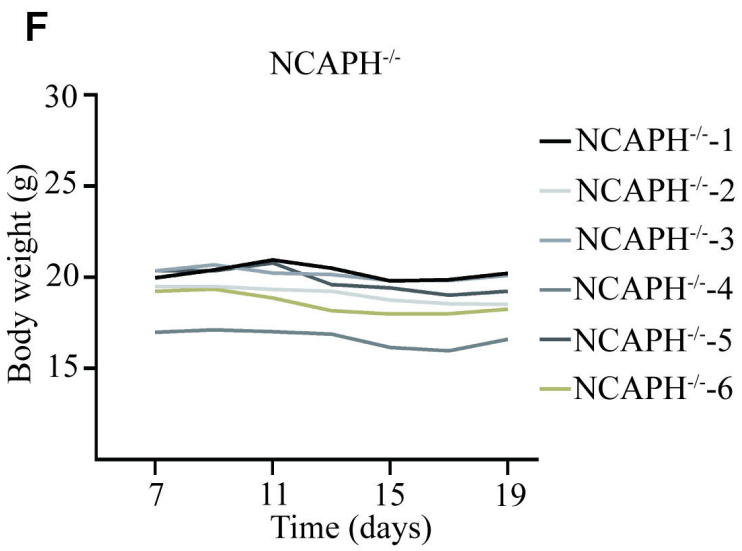

Figure 4. NCAPH knockout inhibited tumor growth in vivo. A) Illustration of the experimental design. B) Images of mice bearing tumors from WT or NCAPH knockout MKN45 cells on day 19. C, D) Tumor volume and weight of WT and NCAPH knockout MKN45 cell xenografts in mice. E, F) NCAPH knockout had no significant effect on the bodyweight of mice. 


\section{Discussion}

GC is one of the most common devastating and deadly malignancies worldwide, ranking fifth in terms of cancer incidence and third in terms of cancer-associated mortality [3]. Condensins are multiprotein complexes that play a crucial role during mitosis, meiosis, and tumorigenesis as chromosome regulators. Recent studies have shown that condensin subunits have important roles in the condensation and decompensation of chromosomes during the cell cycle [17]. NCAPH is one of the three non-SMC subunits in condensin I and is upregulated in multiple cancers [14]. However, the functions of the NCAPH in GC have not been well explored. In this study, we found that NCAPH is overexpressed in various human tumors according to the Oncomine database analysis. Furthermore, we found that NCAPH expression is higher in GC cells than in normal gastric cells. Moreover, the immunohistochemical staining results showed that the expression of NCAPH is clearly increased in GC tissues compared with paracarcinoma tissues. According to the results, NCAPH plays a critical role in the progression of GC.

Normal cells undergo chromosome replication, cell growth, division, and the cell cycle. Abnormal cell proliferation is one of the characteristics of cancer. Thus, cell cycle dysregulation and carcinogenesis are strongly associated with tumor progression [18]. NCAPH is presumed to be a protein that participates in sister chromatid separation during mitosis and plays a critical role in the cell cycle [10]. Existing evidence indicates that the NCAPH gene is involved in the regulation of mitosis. Currently, NCAPH has been confirmed to be involved in GC progression by impacting cell proliferation, migration, invasion, and apoptosis [19]. Previous studies also indicated that NCAPH influenced the DNA damage response [13]. Our study showed through CCK-8 and colony formation assays that knocking out NCAPH significantly inhibited proliferation and colony formation assays in the AGS and HGC27 cell lines. Furthermore, we analyzed the cell migration and invasion by wound healing and Transwell assays. The results showed that NCAPH knockout suppressed AGS and HGC27 cell migration and invasion. In addition, the flow cytometry assay demonstrated that knockout of NCAPH induced the G1 phase cell cycle arrest. Furthermore, the studies showed that the knockout of NCAPH significantly inhibited the expression of proteins related to the cell cycle and DNA damage repair response in AGS and HGC27 cells. NCAPH knockout also significantly inhibited tumor growth in vivo. Therefore, the suppression of proliferation in AGS and HGC27 cells with NCAPH knockout is likely caused by DNA replication defects. These data suggested that NCAPH is a critical oncogenic factor of cell proliferation in vitro and in vivo.

As previously reported [20-22], condensins play an important role in the cell cycle, and cell cycle arrest is one of the mechanisms that inhibits tumor growth. To explore the regulatory effect of NCAPH in GC cells, we performed the gene set enrichment analysis of upregulated genes, which showed that NCAPH was mainly enriched in the cell cycle and DNA replication. We showed that the knockout of NCAPH significantly inhibited the proteins related to the cell cycle. The DNA damage response is an important pathway that is involved in the regulation of cell proliferation, metastasis, and invasion. To further elucidate the regulatory effect of NCAPH in GC cells, we assessed the effects of NCAPH on the expression levels of DNA damage repair proteins in AGS and HGC27 cells by western blot analysis. The results showed that knocking out NCAPH expression decreased the protein levels of DNA damage response markers. In contrast, knocking out NCAPH in AGS and HGC27 cells led to significantly reduced expression of DNA damage repair proteins. Furthermore, the results showed that NCAPH localized to the nucleus, and knockout promoted AGS cell apoptosis and suppressed the expression of EMT-related proteins in AGS cells and HGC27 cells. We speculate that NCAPH knockout can cause DNA replication damage, leading to cell cycle arrest. This indicates that knocking out NCAPH inhibits cell proliferation and migration by cell cycle arrest and DNA damage response. However, the detailed mechanism by which NCAPH modulates the damage repair response needs to be further explored.

In conclusion, our study indicates that NCAPH is upregulated in GC cells and that NCAPH knockout suppresses GC cell migration and invasion in vitro and tumor growth in vivo. Thus, NCAPH might be a tumor oncogene that promotes GC proliferation. NCAPH could be a novel diagnostic marker of the progression of GC and a potential therapeutic target in the future.

Acknowledgments: This work was supported by grants from Cuiying Scientific and Technological Innovation Program of Lanzhou University Second Hospital (No. CY2017-QN18), Lanzhou Science and Technology Fund Program (No. 2018-1-111), Lanzhou Cheng-Guan District Science and Technology Fund Program (No. 2019SHFZ0023), and Lanzhou Science and Technology program project funds (No. 2018-3-47).

\section{References}

[1] GOLLAVILLI PN, PARMA B, SIDDIQUI A, YANG H, RAMESH V et al. The role of $\mathrm{miR}-200 \mathrm{~b} / \mathrm{c}$ in balancing EMT and proliferation revealed by an activity reporter. Oncogene 2021; 40: 2309-2322. https://doi.org/10.1038/s41388-02101708-6

[2] YU ZY, JIANG XY, ZHAO RR, QIN JJ, LUO CJ et al. Effect of KIF22 on promoting proliferation and migration of gastric cancer cells via MAPK-ERK pathways. Chin Med J (Engl) 2020; 133: 919-928. https://doi.org/10.1097/ cm9.0000000000000742

[3] LIU AR, HE QS, WU WH, DU JL, KUO ZC et al. Body composition and risk of gastric cancer: A population-based prospective cohort study. Cancer Med 2021; 10: 2164-2174. https://doi.org/10.1002/cam4.3808 
[4] CHEN W, ZHENG R, BAADE PD, ZHANG S, ZENG H et al. Cancer statistics in China, 2015. CA Cancer J Clin 2016; 66: 115-132. https://doi.org/10.3322/caac.21338

[5] TORRE LA, BRAY F, SIEGEL RL, FERLAY J, LORTETTIEULENT J et al. Global cancer statistics, 2012. CA Cancer J Clin 2015; 65: 87-108. https://doi.org/10.3322/caac.21262

[6] SOTOUDEH M, SHIRVANI SI, MERAT S, AHMADBEIGI N, NADERI M. MSLN (Mesothelin), ANTXR1 (TEM8), and MUC3A are the potent antigenic targets for CAR T cell therapy of gastric adenocarcinoma. J Cell Biochem 2019; 120: 5010-5017. https://doi.org/10.1002/jcb.27776

[7] HIRANO T. Condensins: universal organizers of chromosomes with diverse functions. Genes Dev 2012; 26: 16591678. https://doi.org/10.1101/gad.194746.112

[8] GREEN LC, KALITSIS P, CHANG TM, CIPETIC M, KIM $\mathrm{JH}$ et al. Contrasting roles of condensin I and condensin II in mitotic chromosome formation. J Cell Sci 2012; 125: 15911604. https://doi.org/ 10.1242/jcs.097790

[9] SHINTOMI K, HIRANO T. The relative ratio of condensin I to II determines chromosome shapes. Genes Dev 2011; 25: 1464-1469. https://doi.org/10.1101/gad.2060311

[10] WANG M, QIAO X, COOPER T, PAN W, LIU L et al. HPV E7-mediated NCAPH ectopic expression regulates the carcinogenesis of cervical carcinoma via PI3K/AKT/SGK pathway. Cell Death Dis 2020; 11: 1049. https://doi.org/10.1038/ s41419-020-03244-9

[11] WOOD AJ, SEVERSON AF, MEYER BJ. Condensin and cohesin complexity: the expanding repertoire of functions. Nat Rev Genet 2010; 11: 391-404. https://doi.org/10.1038/ nrg2794

[12] CHEN X, ZHANG D, JIANG F, SHEN Y, LI X et al. Prognostic Prediction Using a Stemness Index-Related Signature in a Cohort of Gastric Cancer. Front Mol Biosci 2020; 7: 570702. https://doi.org/10.3389/fmolb.2020.570702

[13] KIM JH, YOUN Y, KIM KT, JANG G, HWANG JH. NonSMC condensin I complex subunit $\mathrm{H}$ mediates mature chromosome condensation and DNA damage in pancreatic cancer cells. Sci Rep 2019; 9: 17889. https://doi.org/10.1038/ s41598-019-54478-3
[14] YIN L, JIANG LP, SHEN QS, XIONG QX, ZHUO X et al. NCAPH plays important roles in human colon cancer. Cell Death Dis 2017; 8: e2680. https://doi.org/10.1038/cddis.2017.88

[15] XIONG YC, WANG J, CHENG Y, ZHANG XY, YE XQ. Overexpression of MYBL2 promotes proliferation and migration of non-small-cell lung cancer via upregulating NCAPH. Mol Cell Biochem 2020; 468: 185-193. https://doi. org/10.1007/s11010-020-03721-x

[16] GIBSON DG, YOUNG L, CHUANG RY, VENTER JC, HUTCHISON CA $3^{\text {RD }}$ et al. Enzymatic assembly of DNA molecules up to several hundred kilobases. Nat Methods 2009; 6: 343-345. https://doi.org/10.1038/nmeth.1318

[17] WANG HZ, YANG SH, LI GY, CAO X. Subunits of human condensins are potential therapeutic targets for cancers. Cell Div 2018; 13: 2. https://doi.org/10.1186/s13008-018-0035-3

[18] OTTO T, SICINSKI P. Cell cycle proteins as promising targets in cancer therapy. Nat Rev Cancer 2017; 17: 93-115. https://doi.org/10.1038/nrc.2016.138

[19] KIM B, KIM SW, LIM JY, PARK SJ. NCAPH Is Required for Proliferation, Migration and Invasion of Non-small-cell Lung Cancer Cells. Anticancer Res 2020; 40: 3239-3246. https://doi.org/10.21873/anticanres.14305

[20] BATTY P, GERLICH DW. Mitotic Chromosome Mechanics: How Cells Segregate Their Genome. Trends Cell Biol 2019; 29: 717-726. https://doi.org/ 10.1016/j.tcb.2019.05.007

[21] WARD JR, VASU K, DEUTSCHMAN E, HALAWANI D, LARSON PA et al. Condensin II and GAIT complexes cooperate to restrict LINE-1 retrotransposition in epithelial cells. PLoS Genet 2017; 13: e1007051. https://doi.org/10.1371/ journal.pgen.1007051

[22] ABRAMO K, VALTON AL, VENEV SV, OZADAM H, FOX AN et al. A chromosome folding intermediate at the condensin-to-cohesin transition during telophase. Nat Cell Biol 2019; 21: 1393-1402. https://doi.org/10.1038/s41556019-0406-2 